Vidyodaya J. of Sci., (1992) Vol. 4, No. 1, pp: $131-136$

\title{
A PRELIMINARY STUDY OF SATELLITE MAGNETIC ANOMALIES OVER SRI LANKA AND NEARBY OCEANIC REGIONS.
}

\author{
D. A. Tantrigoda and P. Geekiyanage \\ Department of Physics, \\ University of Sri Jayewardenepura, \\ Nugegoda, Sri Lanka
}

Received on: $17-06-1992$

Accepted on: 09-08-1992

\begin{abstract}
Satellite magnetic anomalies over Sri Lanka and nearby oceanic regions are inverted to obtain the crustal magnetization distribution of the region using the least-squares technique and the eigen-value decompostion method. Correlation between the major geological features and the magnetization distribution is also discussed.
\end{abstract}

Key words: Satellite Magnetic Anomalies.

\section{Introduction}

Careful analysis of COSMOS 49, OGO 2,4 and 6 (POGO) satellite magnetic data by the U.S. Geological Survey and NASA led to the identification of magnetic anomalies of crustal origin. One striking example of such an anomaly is the Bangui magnetic anomaly over Central Africa (Regan and Marsh, 1982). Separation of the crustal contribution from satellite magnetic anomalies is a difficult task as this is less than $0.05 \%$ of the main field $(40,000 \mathrm{nT})$ and also is superimposed on approximately ten times larger ionospheric noise. Regan and Marsh (1982) developed approximate techniques for this purpose and isolated the componet of crustal origin from POGO data and compiled a global stellite magnetic anomaly map (between $\pm 50^{\circ}$ latitude). This map gives $1^{\circ}$ averaged magnetic scalar anomalies at a mean altitude of $540 \mathrm{~km}$.

The MAGSAT mission, which was launcheed in October 1979, measured both scalar and vector components of the Earth's magnetic field at a lower altitude (Langel et al., 1984). Several workers have used both POGO and MAGSAT data to investigate the large scale magnetization distribution of the crust (Mayhew, 1979: Mayhew and Johnson, 1987: and Harrison et al., 1986). The technique which has often been used for this purpose is the equivalent source method first introduced by Mayhew (1979). This involves the mathematical representation of crustal magnetic moments by an array of dipoles oriented in the direction of the main field on the surface of the Earth 
and the inversion of observed data to determine the magnetic moments of the dipoles of the array using a suitable method such as the least-squares technique. In this method, it is assumed that the large scale magnetization of the Earth's crust is only by induction.

This paper presents an interpretation of satellite magnetic anomalies over Sri Lanka and surrounding oceanic regions using the equivalent source technique. The correlation between the major geological features and the magnetization distribution obtained from the interpretation is also briefly discussed.

\section{Data Analysis and the Method}

Satellite magnetic anomaly map over India and nearby regions compiled by Agarwal et al. (1986) used in the interpretation presented in this paper. This map has been prepared by using MAGSAT data having $K_{p} \leqslant 3$. The crustal component of the magnetic field was obtained by removing from the observations main field values, estimated by MAGSAT (4/81) from the 14th degree and order spherical harmonic expansion, and the contribution due to ionospheric ring currents, approximated by the Legendra polynomial of degree one. A secondary long wavelength ring current correction was also carried out by fitting a quadratic function to each profile of the map. Finally a running three point weighted average was applied to each profile in order to remove high frequency instrumental noise.

Interpretation of satellite magnetic anomalies over Sri Lanka and surrounding Indian ocean region was carried out using the equivalent source technique as indicated in the introduction. In this method, it is assumed that there is no large scale remanent magnetization of the crust and that the induced magnetization can be represented by a series of dipoles placed on the surface of the Earth along the direction of the Earth's main field. The magnetic anomaly values obtained after the above-mentioned corrections were inverted using the least-squares technique to determine the magnetic dipole moment of each dipole in the array. It was observed that the results of the inversion depended on the spacing of dipoles on the surface of the Earth. The magnetic moment distribution was highly unstable (ie. fluctuated between large positive and negative values) for certain values of spacing between the dipoles. Sucn a set of values does not represent a physically possible magnetization distribution although it is mathematically correct.

The correlation between the observed anomaly and the anomaly calculated starting from the magnetic moments obtained from the inversion gives the measure of "fit" between observed and calculated anomalies. The standard deviation of the magnetic moments calculated gives a measure of the stability of the solution. It was found that there is a reasonable trade off between the stability and the "fit" of observed and calculated anomalies when the 
spacing between the dipoles is $215 \mathrm{~km}$. Even at this value of spacing, calculated magnetization distribution shows a "bull's eye" pattern having no physical meaning.

Langel et al. (1984) have shown that inversion of satellite magnetic data at latitudes less than $20^{\circ}$ gives results which are unstable for any value of dipole spacing. They have recommended the use of the principal value decomposition method when the dipoles are situated at latitudes less than $\pm 20^{\circ}$.

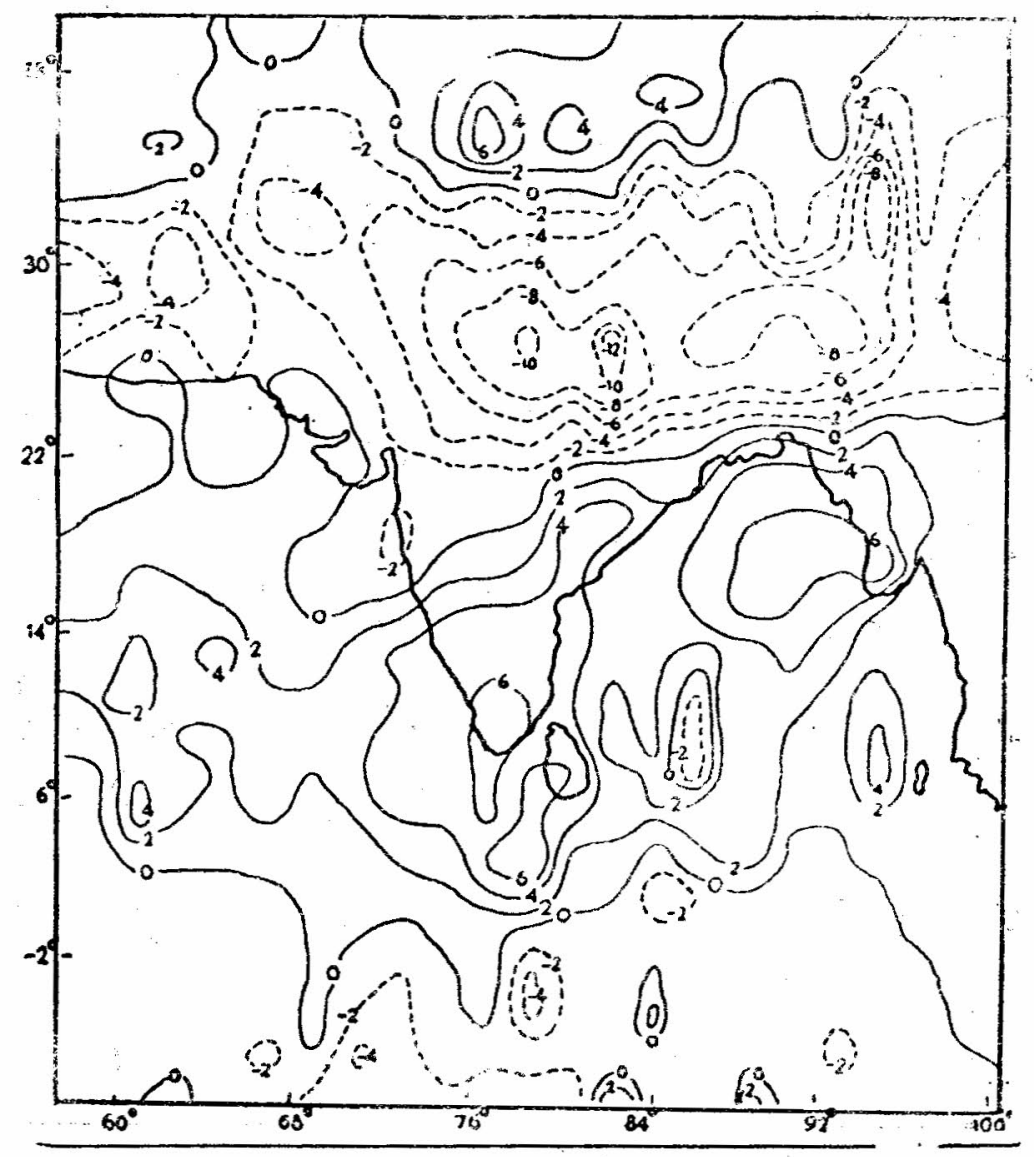

Fig. 1. Anomaly in total field (B) for average MAGSAT heights (after Agarwal et al., 1986). Contours are in nT.

\section{Results and Discussion}

Fig. 1 shows the satellite magnetic anomaly map over Sri Lanka and nearby regions used in this study. The various corrections discussed above were first carried out on this map. Then the anomaly values were inverted using the least-squares technique to find the moments of an array of dipoles situated over the region under consideration. The spacing of dipoles was 
$215 \mathrm{~km}$. The magnetization distribution of the region was calculated assuming each dipole represents a rectangular prismatic section of the crust having dimensions $215 \times 215 \times 40 \mathrm{~km}^{3}$ (it is assumed that the thickness of the crust is $40 \mathrm{~km}$ ). Fig. 2 depicts the magnetization distribution obtained from the calculation in the form of a contour map. As can be seen from the figure, magnetization values fluctuate between negative and positive values having no physical meaning. Therefore the anomaly map was inverted again using the principal value decomposition method. As in the previous case, magnetic dipole values were converted into the magnetization distribution of the crust using the same assumption and the results are shown in Fig. 3.

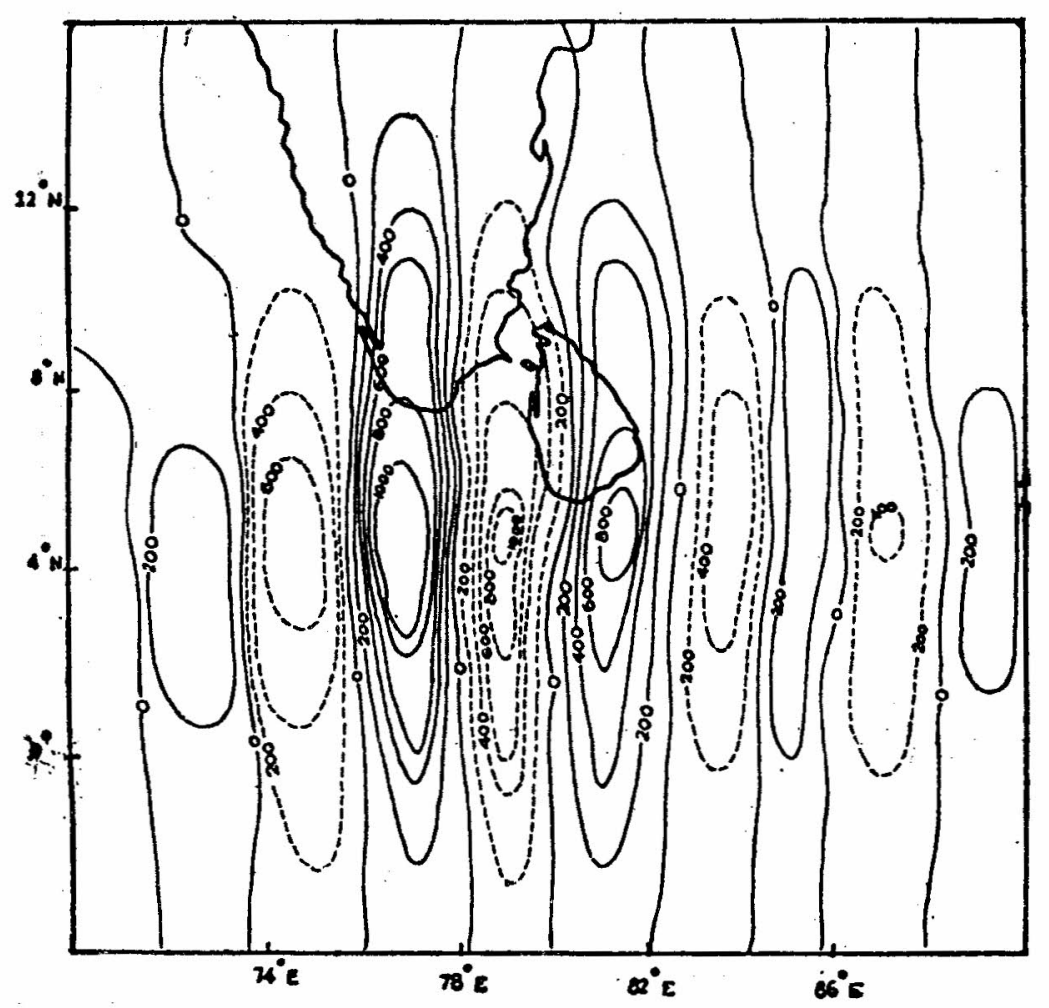

Fig. 2 Crustal magnetization distribution of Sri Lanka and contiguous regions (in $2 \times 10^{-4} \mathrm{Am}^{-1}$ ) obtained from the least-square inversion. 


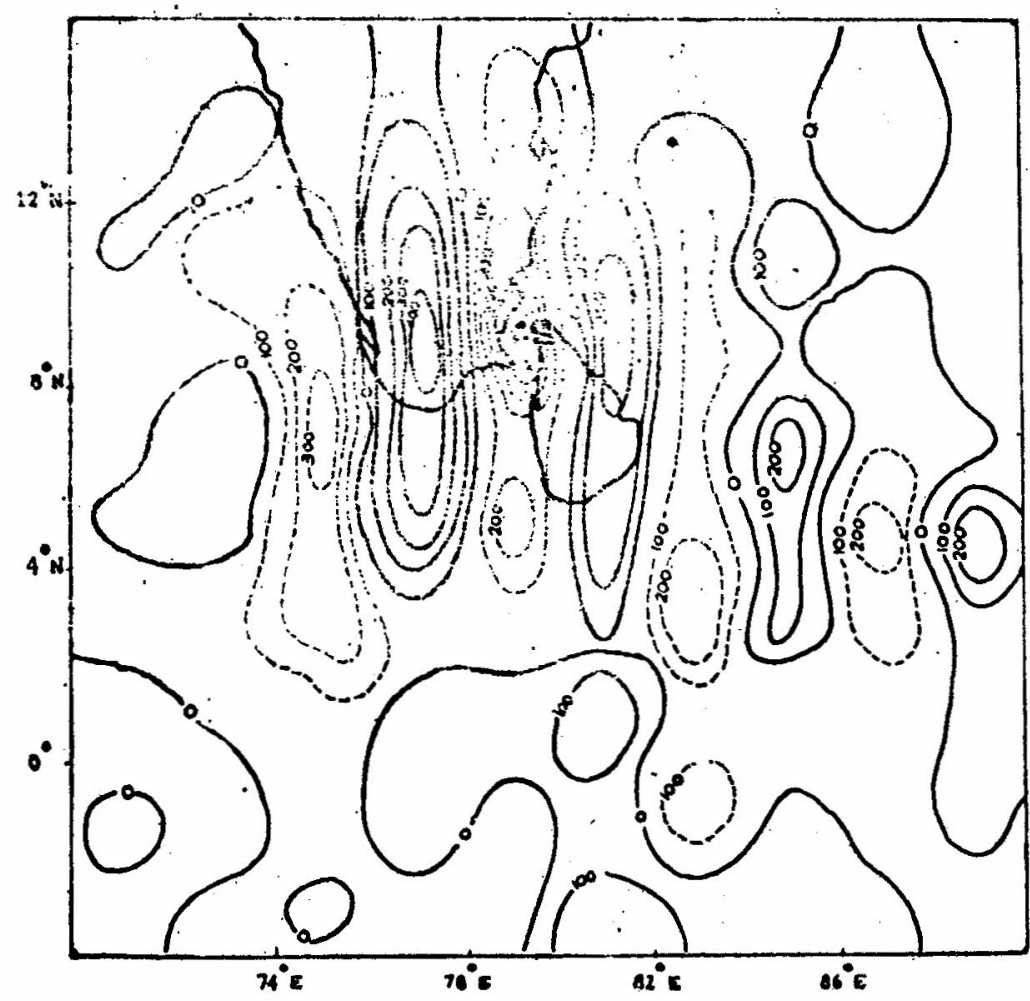

Fig. 3 Crustal magnetization distribution of Sri Lanka and contiguous regions (in $2 \times 10^{-4} \mathrm{Am}^{-1}$ ) obtained from the eigen value decompositon method.

Fig. 3 shows regions of positive magnetization as well as negative magnetization. It should be noted that we have assumed at the outset that there is no large scale remanent magnetization in the crust. Therefore, negative and positive magnetization values of Fig. 3 do not represent reverse and normal magnetization of the crust. However, we can conclude that positive magnetization values imply high magnetization of the curst and the negative magnetization values imply low magnetization.

As can be seen from Fig. 3, the magnetization of Sri Lankan rocks changes from positive to negative across a certain line. This implies that the magnetization changes from strong to weak over this line. Further, this line more or less coincides with the eastern Highland-Vijayan boundary which separates two major geological units of Sri Lanka. This indicates the presence of more magnetized basic rocks underneath the Vijayan side of the boundary compared to the Highland side of the boundary. 
Another interesting feature of the magnetization distribution map is a set of very low magnetization values over the Palk strait. This may be due to a thermal anomaly in the area. If the heat flow values are comparatively high over this area, then the magnetization values should become low as the high temperatures can disturb the orientation of magnetic elements along the direction of the Earth's field.

\section{References}

Agarwal, A.K., Singh, B.P., Rastogi, R G. and"Srinivasan, S.. 1986 On utility of spaceborne vector magnetic measurements in crustal studies, Phys. Earth Planet. Inter., $41,260-268$.

Harrison, C.G.A., Carle, H.M. and Hayling. K.L., 1986. Interpretation of satellite elevation magnetic anomalies, J. Geophys. Res. 91, 3633-3650.

Langel, R.A., Slud, E.V. and Smith, P.J., 1984. Reduction of satellite magnetic anomaly daia, J Geophys. 54, 207-212

Mayhew, M.A., 1979. Inversion of satellite magnetic anomaly data, J. Geophys. $45, \quad 119-128$.

Mayhew, M.A., and Johnson, B.D., 198i. An equivalent layer magnetization model for Australia based on MAGSAT data, Earth Planet Sci Lett 83, 167-174.

Regan, R.D., and Marsh, B..D.. 1982. The Bangui magnetic anomaly: its geological origin, J. Geophys. Res. 87, 1107-1120. 